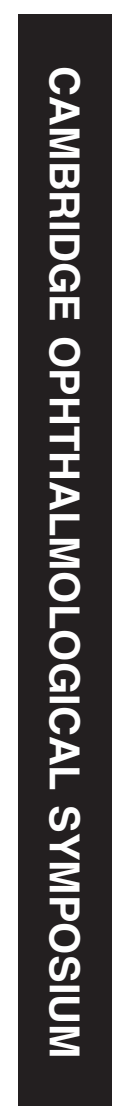

${ }^{1}$ Department of Ophthalmology, BC Children's Hospital, Vancouver, British Columbia, Canada

${ }^{2}$ Department of Ophthalmology and Visual Sciences, University of British Columbia, Vancouver, British

Columbia, Canada

Correspondence: CJ Lyons, Department of Ophthalmology, BC Children's Hospital, Room A136, 4480 Oak Street, Vancouver, British Columbia V6H 3V4, Canada

Tel: +1 604875 3117;

Fax: +1 6048753561 .

E-mail: cjlyons@telus.net

${ }^{3}$ These authors contributed equally to this work.

Received: 15 October 2014 Accepted: 24 October 2014 Published online: 9 January 2015

\section{Cranial nerve palsies in childhood}

\begin{abstract}
We review ocular motor cranial nerve palsies in childhood and highlight many of the features that differentiate these from their occurrence in adulthood. The clinical characteristics of cranial nerve palsies in childhood are affected by the child's impressive ability to repair and regenerate after injury. Thus, aberrant regeneration is very common after congenital III palsy; Duane syndrome, the result of early repair after congenital VI palsy, is invariably associated with retraction of the globe in adduction related to the innervation of the lateral rectus by the III nerve causing cocontraction in adduction. Clinical features that may be of concern in adulthood may not be relevant in childhood; whereas the presence of mydriasis in III palsy suggests a compressive aetiology in adults, this is not the case in children. However, the frequency of associated CNS abnormalities in III palsy and the risk of tumour in VI palsy can be indications for early neuroimaging depending on presenting features elicited through a careful history and clinical examination. The latter should include the neighbouring cranial nerves. We discuss the impact of our evolving knowledge of congenital cranial dysinnervation syndromes on this field.
\end{abstract}

Eye (2015) 29, 246-251; doi:10.1038/eye.2014.292; published online 9 January 2015

\section{Introduction}

Palsies of cranial nerves III, IV, and VI are rare in childhood, with an estimated incidence of 7.6 per $100000 .{ }^{1}$ The affected child differs from the adult in several ways. In the presence of cranial nerve paresis, the child's developing visual system is liable to develop strabismic, deprivation, or anisometropic amblyopia. Fusion and stereopsis may be reduced or abolished. The child also differs from the adult in his/her impressive ability for repair, altering the clinical presentation of cranial nerve paresis in childhood. In this brief review of cranial nerve paresis in childhood we highlight some of these differences.

We previously reported our experience with IIIrd nerve palsy in children, ${ }^{2}$ a series of 18 children presenting under 15 years of age. The causes are shown in Table 1. As in other larger pediatric series, ${ }^{3}$ congenital and trauma were the most common aetiological factors. The frequency of associated neurological abnormalities (arachnoid cyst, optic nerve hypoplasia, and so on) supported the recommendation of neuroimaging of all children presenting with congenital or acquired cranial nerve III palsy. Notable features in our series were the almost universal presence of aberrant regeneration because of misrouting of regenerating III fibres in congenital palsy (5 of $6(83 \%)$ ) and the frequent presence of pupil involvement that, in contrast to the adult, did not suggest an increased likelihood of a compressive aetiology. As in Adie's pupil, symptomatic mydriasis can be treated with Pilocarpine $0.1 \%$ drops in the affected eye. In addition, like Adie's pupil, the mydriatic pupil of III palsy is frequently observed to become miotic, often over a period of years.

Congenital III palsy may present with complete ptosis and ophthalmoplegia or a partial palsy with or without aberrant regeneration. The degree of paralysis and subsequent recovery determines the likelihood of achieving a reasonable visual acuity in the affected eye; satisfactory alignment with the use of an anomalous head posture may be associated with a good visual outcome. This was the case in 6 of our patients (33\%). Other important amblyogenic factors include reduced accommodation in the affected eye. In the presence of underlying hypermetropia, so common in children, this is equivalent to penalization of the affected eye. Correction of the hypermetropia and use of a bifocal lens for 
Table 1 Causes of III palsy in childhood

\begin{tabular}{lcc}
\hline & $N g$ and Lyons $^{2}$ & Biglan and colleagues $^{3}$ \\
\hline Congenital & $33 \%$ & $38 \%$ \\
Trauma & $28 \%$ & $32 \%$ \\
Tumour & $22 \%$ & $11 \%$ \\
Vascular & $11 \%$ & $11 \%$ \\
Meningitis & $6 \%$ & \\
Idiopathic & $2 \%$ & \\
\hline
\end{tabular}

$\mathrm{Ng}$ and Lyons ${ }^{2}$ and percentages from Biglan series. ${ }^{3}$

near is helpful, along with an appropriate occlusion regime for the contralateral eye.

A congenital or acquired III palsy with subsequent repair and aberrant regeneration should be distinguished from the much more worrying history of a previously normal child who has slowly (often over a period of years) developed a III palsy, with progressive ptosis and exotropia (with or without pupil signs) but who has evidence of aberrant regeneration. The temporal pattern of this 'primary aberrant regeneration' should alert the clinician to the possibility of nerve damage from a compressive process with simultaneous ongoing repair. This is a classical sign of a slow-growing lesion in the cavernous sinus such as a schwannoma or meningioma. ${ }^{4}$ The corneal sensation may also be decreased indicating concurrent $V$ th nerve involvement. This clinical scenario should prompt magnetic resonance imaging (MRI) of the brain with particular attention to the cavernous sinus.

Neurofibromatosis 2, a tumour-suppressor mutation, has a relative predilection for tumour formation in cranial nerves III and V, probably because of their relative bulk compared with cranial nerves IV and VI.

Rarely, repair does not result in resolution of III palsy with or without aberrant regeneration. Instead, children with oculomotor nerve paresis with cyclic spasms have a history of unilateral congenital III palsy with ptosis, esotropia, and mydriasis. Every 2 min or so, bursts of III function result in elevation of the ptotic eyelid as the globe starts to adduct and the pupil constricts. This 'spastic phase' lasts $\sim 30 \mathrm{~s}$ before III function reverts to the 'paretic phase'. ${ }^{5}$ This condition, sometimes also known as 'Cyclical III palsy', likely results from disrupted repair processes following congenital III; Miller ${ }^{6}$ demonstrated very similar findings in two adults who had previously undergone skull-base irradiation with subsequent repair, a process that the author felt was similar to the development of neuromyotonia.

In recent years, there has been a rapid evolution in our understanding of the disorders formerly known as congenital fibrosis of the extraocular muscles. These disorders, identified as CFEOM 1-3, resulted in variable manifestations of ptosis with vertical movement deficits and reduction of horizontal eye movements. Pupillary signs are occasionally evident as these disorders reflect variable congenital involvement of the III nerve. We examined an affected infant under general anaesthesia at the age of 10 months and noted free forced duction testing. The same examination repeated in this patient at the age of 9 years showed marked restriction, particularly in elevation. 'Congenital Fibrosis' is thus a misnomer as the restrictive 'fibrosis' is a feature that develops secondary to disuse related to primary congenital dysinnervation.

The phenotypic patterns were named CFEOM 1-3, but as is often the case in pediatric ophthalmology, the underlying genetic mutations that arise anywhere from the brainstem to the orbit are forcing a rethink of this group of conditions that are now referred to as the congenital cranial dysinnervation disorders (CCDDs) ${ }^{7,8}$

Fourth (trochlear) nerve palsy is the commonest congenital cranial nerve palsy. ${ }^{1}$ Physically slender, with a long intracranial course, this nerve is subject to traumatic damage. Unilateral or bilateral fourth nerve palsy results in characteristic motility patterns including elevation in adduction, V-pattern related to reduced abduction force in downgaze with unopposed adduction by the inferior recti, and excyclotorsion. Chin-down head posture for bilateral and contralateral head tilt for unilateral palsy are noted, and the diagnosis is made clinically with support from orthoptic testing that reveals large vertical fusional ranges for long-standing or congenital cases. Thus, neuroimaging is rarely indicated.

However, several imitators exist. In particular, the craniofacial disorders are known to produce ocular motility patterns that are virtually identical. Some patients may also present with this characteristic ocular motility pattern without overt craniofacial signs (Figure 1). In the presence of an unexplained primary V-pattern with bilateral superior oblique underaction or, conversely, a primary A-pattern with bilateral superior oblique overaction, we recommend a careful examination of the lid fissure, as a marked mongoloid or antimongoloid slant may be the only indicator of underlying ex- or in-cyclo rotation of the orbital contents as seen in craniofacial disorders. An accompanying oblique astigmatism may confirm this entity. Coronal cuts on orbital MRI will highlight the lateral displacement of the superior recti and medial displacement of the inferior recti, superior displacement of the medial recti, and inferior shift of the lateral recti, emphasizing the orbital cause of this primary V-pattern ocular motility disorder. An analogous condition has been described with primary A-pattern with incyclotorsion of the orbital contents. ${ }^{9}$

Fourth nerve palsy imitations frequently go undetected. Occasionally a Superior oblique tendon is 
a
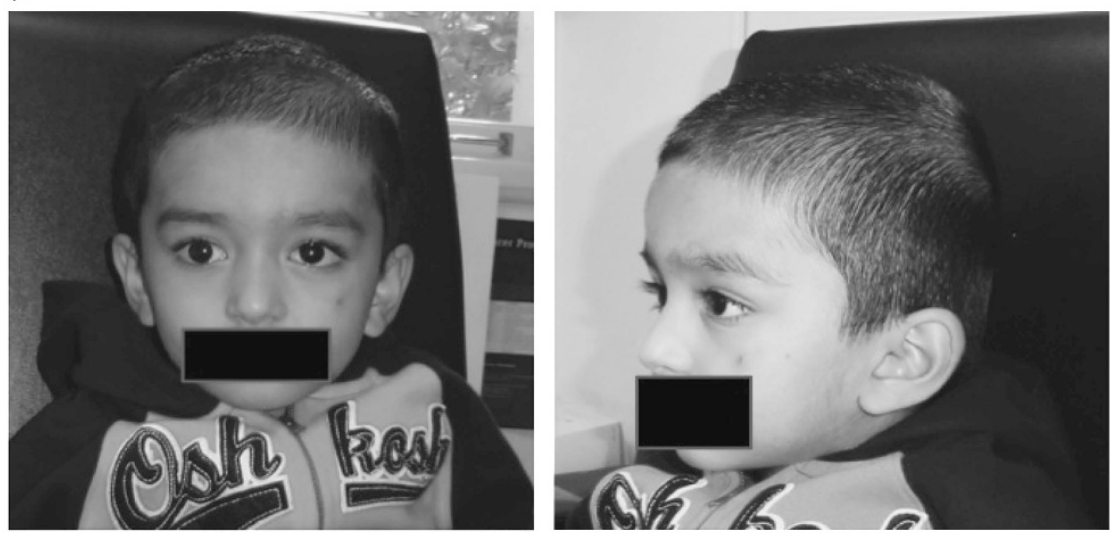

b
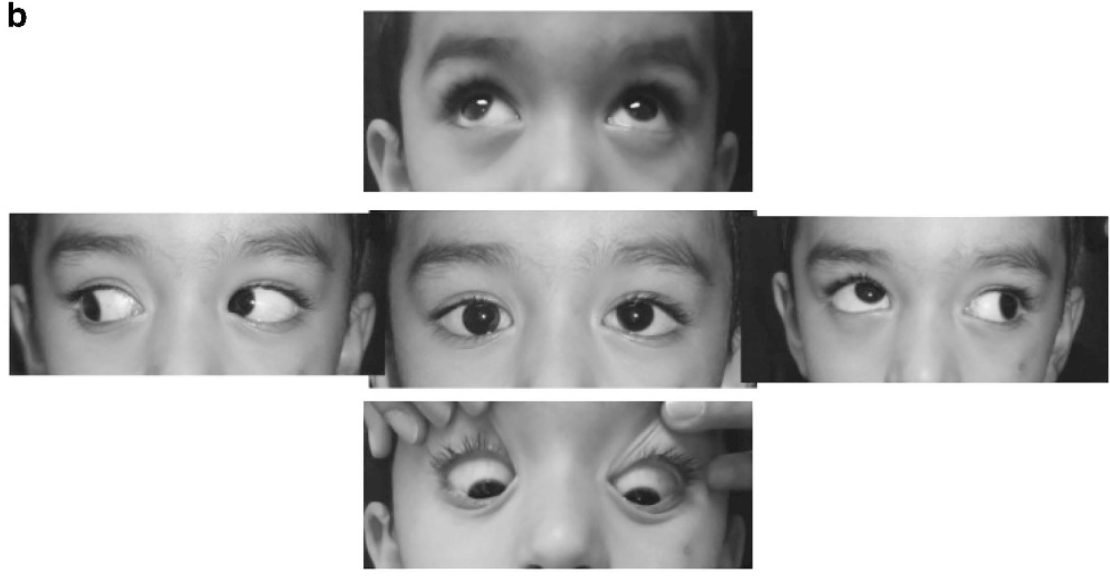

C

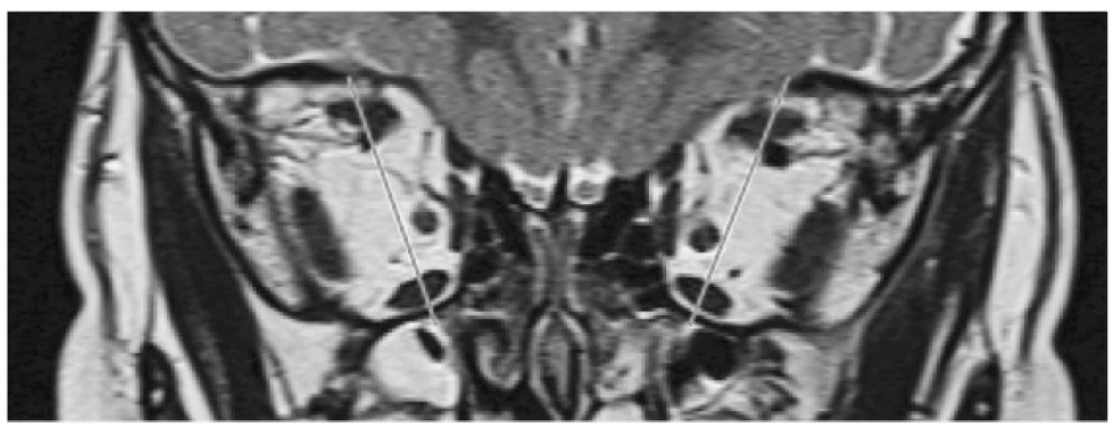

Figure 1 (a) This 3-year-old boy does not have any facial features of craniofacial synostosis. (b) He presents with a V-pattern exotropia with marked elevation of each eye in adduction, suggesting a diagnosis of bilateral superior oblique palsy. (c) Coronal T2-weighted MRI shows excyclorotation of the orbital contents. Lines are drawn through the centre of the vertical recti on each side emphasizing the muscle displacement. There is un-pairing of the horizontal yoke muscles, leading to elevation of the adducting eye and depression of the abducting eye on each side. A full colour version of this figure is available at the Eye journal online.

found at surgery to be completely absent. ${ }^{10}$ In the past few years, radiological techniques using higher-resolution 3T MRI have been used to identify the fourth nerve in normal patients and those with congenital fourth nerve palsy. ${ }^{11}$ A total of 97 patients with a history consistent with congenital IV nerve palsy were reviewed: the ipsilateral IVth nerve was absent in $73 \%$ of patients, with variable ipsilateral superior oblique hypoplasia.
The authors suggested that congenital IVth nerve palsy, a common and unexplained form of congenital cranial nerve paresis, was also a form of CCDD.

$\mathrm{Knapp}^{12}$ wrote a classic series of papers regarding the surgical management of IVth nerve palsy, emphasizing the importance of careful preoperative measurements, tailoring surgery to the gaze direction in which the deviation is greatest through judicious use of rectus 
Table 2 Causes of VI nerve palsy in a series of 75 children who had undergone neuroimaging (Lee $\mathrm{et}_{\mathrm{al}} \mathrm{l}^{14}$ )

\begin{tabular}{ll}
\hline Neoplasm & $45 \%$ \\
Raised ICP & $15 \%$ \\
Trauma & $12 \%$ \\
Congenital & $11 \%$ \\
Inflammatory & $7 \%$ \\
Idiopathic, miscellaneous & $10 \%$ \\
\hline
\end{tabular}

recessions, inferior oblique weakening, and superior oblique tuck.

Fells and his team ${ }^{13}$ reviewed the management of bilateral superior oblique palsy and stressed the importance of dealing with any significant torsion as a primary procedure, using his modification of the Harada Ito procedure. Failure to deal with the torsion was an important barrier to a satisfactory fusion result in this series. Identification of masked bilaterality and appropriate planning for torsional correction are important in preoperative surgical planning for bilateral IVth nerve palsy.

Lee et $a l^{14}$ reported a series of 75 children with VIth nerve palsy, all of whom had undergone neuroimaging. (Table 2) They found that almost half of the cases were due to neoplasm and suggested that neuroimaging should be considered. Conversely, Holmes et al in their population-based review of cranial nerve palsies in children found that no case of intracranial neoplasia presented as an isolated cranial nerve palsy. We recommend neuroimaging for the majority of cases, particularly if there is involvement of the neighbouring cranial nerves. Illustrative patients (Figures 2 and 3) are reminders that the VIth and Vth nerves are contiguous in the cavernous sinus so that loss of corneal sensation indicates pathology at that site. Conversely, the VIth and VIIth nerves are contiguous in the pons and facial nerve weakness accompanying VI palsy points to pontine pathology.

Lee et $a l^{14}$ in their review of 75 children with VIth nerve palsy who had undergone neuroimaging found that $11 \%$ were congenital. In this number, they included children with Duane Syndrome; it is true that Duane and Moebius syndromes, which are both forms of CCDD, are types of congenital VIth nerve paresis; Duane syndrome is a congenital VIth nerve palsy with repair by the IIIrd nerve and Mobius syndrome may present VIth nerve palsy or a full-blown horizontal gaze palsy.

Sixth nerve palsy may complicate otitis media, an association known as Gradenigo syndrome. In addition, there may be venous or even cavernous sinus thrombosis, making this complication of otitis media potentially life-threatening. ${ }^{15}$ It is difficult to know whether the current trend to withhold antibiotics for
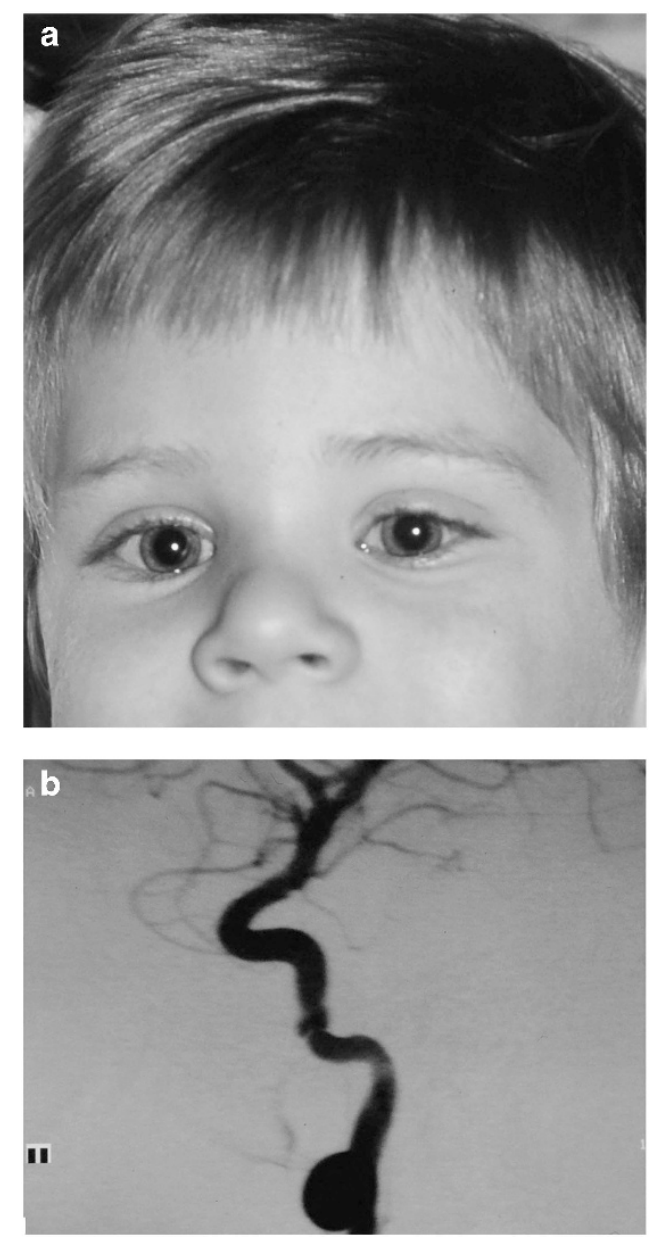

Figure 2 (a) This 3-year-old boy was involved in a severe motor vehicle accident; after a week in intensive care, he was transferred to the ward. At that time he was noted to develop a red left eye, prompting referral to ophthalmology. At $3 \mathrm{~h}$ after referral, the redness had resolved but a left VIth nerve palsy was evident. The ipsilateral corneal sensation was decreased, indicating likely cavernous sinus compression of $\mathrm{V}$ and VI. Neuroimaging and angiography (b) revealed a dissecting carotid aneurysm causing Vth and VIth nerve compression within the cavernous sinus. A full colour version of this figure is available at the Eye journal online.

acute otitis media is contributing to a possible increased incidence of severe complications such as those noted in our report.

Benign idiopathic VIth nerve palsy is a condition that is not uncommon in childhood; Mahoney and Liu ${ }^{16}$ reviewed 225 children with VIth nerve palsies and found that $30(13 \%)$ were in this group. Their mean age was 3 years and 28 of the 30 had an MRI. In four of the patients, the esotropia eventually became comitant. Sturm and Schöffler ${ }^{17}$ reported a group of 12 children followed up for over 9 years. Like previous authors, they pointed out a preponderance of girls affected by a left-sided palsy that resolved in 

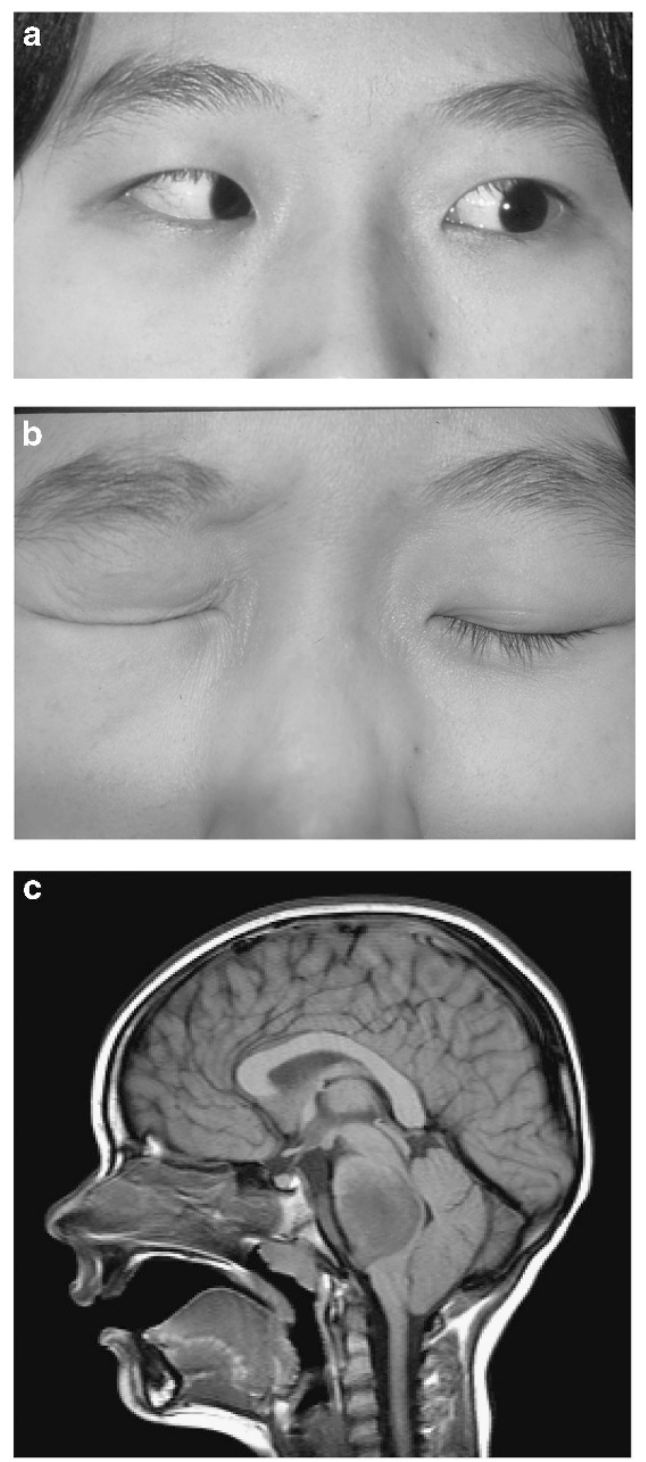

Figure 3 A 17-year-old girl presented with a left VIth nerve palsy (a). Ipsilateral VIIth nerve function was decreased (b). MRI showing a pontine glioma (c). A full colour version of this figure is available at the Eye journal online.

$<6$ months. In three of their patients, they reported an ipsilateral recurrence.

In summary, the clinical characteristics of cranial nerve palsies in childhood are modified by the child's capacity for repair and regeneration after injury. Thus, aberrant regeneration is very common after congenital III palsy; Duane syndrome, a form of congenital VI palsy, is invariably associated with retraction of the globe in adduction related to the innervation of the lateral rectus by the III nerve causing co-contraction in adduction, another form of repair. Clinical features that may be of concern in adulthood may not be relevant in childhood; whereas the presence of mydriasis in III palsy suggests a compressive aetiology in adults, this is not the case in children. However, the frequency of associated CNS abnormalities in III palsy and the risk of tumour in VI palsy can be indications for early neuroimaging depending on presenting features elicited through a careful history and clinical examination. The latter should include the neighbouring cranial nerves.

Recent genetic and neuroradiological evidence, as well as our evolving understanding of the genetic control of the process of extraocular muscle innervation, have highlighted the clinical entity of CCDD. Increasingly, we realize that this group of disorders is an important aetiological factor underlying the congenital cranial nerve palsies.

\section{Conflict of interest}

The authors declare no conflict of interest.

\section{References}

1 Holmes JM, Mutyala S, Maus TL, Grill R, Hodge DO, Gray DT. Pediatric third, fourth, and sixth nerve palsies: a populationbased study. Am J Ophthalmol 1999; 127(4): 388-392.

$2 \mathrm{Ng}$ Ys, Lyons CJ. Oculomotor nerve palsy in childhood. Can J Ophthalmol. 2005; 40(5): 645-653.

3 Schumacher-Feero LA, Yoo KW, Solari FM, Biglan AW. Third cranial nerve palsy in children. Am J Ophthalmol 1999; 128(2): 216-221.

4 Schatz NJ. Primary aberrant aculomotor regeneration. A sign of intracavernous meningioma. Arch Neurol 1977; 34(1): 29-32.

5 Loewenfeld IE, Thompson HS. Oculomotor paresis with cyclic spasms. A critical review of the literature and a new case. Surv Ophthalmol 1975; 20(2): 81-124.

6 Miller NR. Adult-onset acquired oculomotor nerve paresis with cyclic spasms: relationship to ocular neuromyotonia. Am J Ophthalmol 2004; 137(1): 70-76.

7 Chan WM, Andrews C, Dragan L, Fredrick D, Armstrong L, Lyons $\mathrm{C}$ et al. Three novel mutations in KIF21A highlight the importance of the third coiled- coil stalk domain in the etiology of CFEOM1. BMC Genet 2007; 8: 26.

8 Bosley TM, Abu-Amero KK, Oystreck DT. Congenital cranial dysinnervation disorders: a concept in evolution. Curr Opin Ophthalmol 2013; 24: 398-406.

9 Paysse EA, Khokhar A, McCreery KM, Morris MC, Coats DK. Up-slanting palpebral fissures and oblique astigmatism associated with A-pattern strabismus and overdepression in adduction in spina bifida. J AAPOS 2002; 6(6): 354-359.

10 Helveston EM, Giangiacomo JG, Ellis FD. Congenital absence of the superior oblique tendon. Trans Am Ophthalmol Soc 1981; 79: 123-135.

11 Kim JH, Hwang JM. Absence of the trochlear nerve in patients with superior oblique hypoplasia. Ophthalmology 2010; 11711(2208-13): e1-e2.

12 Knapp P. Classification and treatment of superior oblique palsy. Am Orthopt J 1974; 24: 18-22.

13 Price NC, Vickers S, Lee JP, Fells P. The diagnosis and surgical management of acquired bilateral superior oblique palsy. Eye (Lond) 1987; 1(Pt 1): 78-85. 
14 Lee MS, Galetta SL, Volpe NJ, Liu GT. Sixth nerve palsies in children. Pediatr Neurol 1999; 20(1): 49-52.

15 Pollock TJ, Kim P, Sargent MA, Aroichane M, Lyons CJ, Gardiner JA. Ophthalmic complications of otitis media in children. J AAPOS 2011; 15(3): 272-275.
16 Mahoney NR, Liu GT. Benign recurrent sixth (abducens) nerve palsies in children. Arch Dis Child 2009; 94(5): 394-396.

17 Sturm V, Schöffler C. Long-term follow-up of children with benign abducens nerve palsy. Eye (Lond) 2010; 24(1): 74-78. 